\title{
EMCON 2008: 10th Annual National Conference of the Society for Emergency Medicine in India
}

\author{
Vinayaka Mission University, Salem, India, 12-16 November 2008
}

\section{Oral Presentations}

\author{
Tracheostomy tube suctioning: Foley's catheter \\ vs. suction catheter \\ R. Prasad, M. Srinivas, P.N. Pandian, R. Nithyanandham, \\ V.P. Chandrasekaran
}

Vinayaka Mission Kirupananda Variyar Medical College Hospital, Vinayaka Mission University, Salem, India

Background: In the context of today's critical care, many patients require prolonged intubation and tracheostomy care. Though these are life-saving procedures, they are not totally devoid of complications. Suctioning of secretions via the ET tube/tracheostomy tube with a suction catheter is associated with trauma to the passages owing to the structure and material of catheters. To avoid the traumatic complications associated with the suction catheter, we wanted to use Foley's catheter instead of suction catheter (since the former is softer and structurally advantageous than the latter).

Aim: The aim of the study was to compare the advantages of using Foley's catheter vs. suction catheter for suctioning through the endotracheal tube/tracheostomy tube.

Methods: This was a prospective study done over a period of one and half years from Feb 2007 to Aug 2008. All the patients who underwent tracheostomy were included in the study. Patients with bleeding disorders, coagulation profile abnormalities, and ICU stay less than a week were excluded from the study. Every alternate patient was suctioned using Foley's catheter. All the patients were followed till the time of discharge for any complications developed during the study. Data were collected from 363 patients of whom 181 were randomized to Foley's catheter group and 182 to the suction catheter group.

Results: The data obtained from the trial have shown that only $2 \%$ of the patients developed bleeding when Foley's catheter was used as compared to $23 \%$ when the suction catheter was used. Adequate suctioning was obtained in both groups and there was no other significant complication related to the suctioning noted in both methods.

Conclusion: Foley's catheter is superior to conventional suction catheter in preventing tracheal bleed during endotracheal suctioning.

\section{Knowledge of staff nurses on nursing activities to be carried out in an emergency department}

\section{R.N. Krishnan, P. Krishnan, K. Darshan}

Vinayaka Missions Annapoorna College of Nursing, Salem, Tamilnadu, India. darshankutty@yahoo.com

Aim: The aims of the study were to assess the knowledge of staff nurses related to nursing activities to be carried out in an emergency department (ED) and to compare their demographic variables.

Methods: Descriptive cross-sectional survey was done at Vinayaka Mission Hospitals, Salem. Ninety-two staff nurses were selected by convenient sampling technique. Data were collected by using closed ended questionnaire, which consisted of 46 items pertaining to knowledge of staff nurses regarding nursing activities to be carried out in an ED, after testing its validity and reliability $(r=0.99)$.

Results: Demographic characteristics revealed that most of the staff nurses $(89.1 \%)$ were in the age group of 20 25 years, $71.7 \%$ were females and $94.6 \%$ were diploma holders. There were $44.6 \%$ who had $1-3$ years of experience in nursing and around $22 \%$ were from medical ICU and general ward; $61.96 \%$ of them had not attended any in-service programme on nursing activities to be carried out in an emergency department. Amongst the 35 staff nurses who had attended the in-service educational programme, $42.86 \%$ of them attended one to two programmes and $51.44 \%$ of staff nurses attended the last programme $1-3$ years ago. 
The findings on knowledge of nursing activities to be carried out in an ED revealed that most (34.78\%) of staff nurses had average knowledge. Highest mean score (1.94 \pm 1.54 ), which is $48.64 \%$, was obtained by staff nurses in the area 'equipment of ED' and more or less similar mean score $(5.72 \pm 2.12)$ which is $46.47 \%$ was in the area 'records and reports maintained in ED'. Average knowledge and lowest mean score $(4.2 \pm 2.3)$, i.e. $30 \%$ was obtained by staff nurses in the area 'regular nursing activities of ED'. No significant association $(P<0.05)$ was found between knowledge scores of staff nurses in relation to their demographic variables.

Conclusions: From the findings, it can be concluded that level of knowledge of staff nurses regarding nursing activities to be carried out in an ED was inadequate. Hence, it can be suggested to have various educational sessions with practical demonstration based on the learning needs of the staff nurses.

\section{Randomized double-blind placebo-controlled trial comparing room-temperature and heated lidocaine for digital nerve block}

\section{K. Papa, T. V. Ramakrishnan}

Sri Ramachandra Medical Institute, Porur, Chennai, India. kaleemuddinpapa@hotmail.com

\begin{abstract}
Aim: The aim of the study was to determine whether warming of lidocaine decreases pain during injection for digital nerve block.

Methods: A prospective, randomized, double-blind, placebo-controlled trial was done at Sri Ramachandra University Hospital in Chennai India from 1st September 2008 to 31 st October 2008. All patients coming with clear indication of digital nerve block $>18$ years were included. Patients with GCS $<15$ were excluded. Written consent was taken for all patients before administration of nerve block.

Methodology: Patients requiring digital nerve blocks for injuries in fingers and toes were randomly assigned to receive either room-temperature $\left(28^{\circ} \mathrm{C}\right)$ or heated $\left(42^{\circ} \mathrm{C}\right)$ $2 \%$ lidocaine. Blocks were performed in standardized fashion by an emergency physician, who is not a treating doctor. The treating emergency physician was blinded as to which sample was used and rated pain of digital block on a $10-\mathrm{mm}$ visual analog scale (VAS). Efficacy of digital block was tested immediately by VAS score and 5-min VAS score by pin prick and two-point discrimination test. Five-point Likert scale was used for assessing ease of administration of digital nerve block.
\end{abstract}

Results: Fifty patients were included in the study, 17 of which were females.

\begin{tabular}{lll}
\hline & $\begin{array}{l}\text { Lidocaine-room } \\
\text { temperature }\end{array}$ & $\begin{array}{l}\text { Lidocaine-42 }{ }^{\circ} \mathbf{C} \\
\text { temperature }\end{array}$ \\
$\begin{array}{l}\text { No. of subjects } \\
\text { Age (years), mean }\end{array}$ & 25 & 25 \\
$\begin{array}{l}\text { Immediate VAS } \\
\text { score (mean) }\end{array}$ & 7.2 & 29.4 \\
$\begin{array}{c}\text { Immediate VAS } \\
\text { score (median) }\end{array}$ & 7 & 1.92 \\
$\begin{array}{c}\text { 5-min VAS score } \\
\text { (mean) }\end{array}$ & 0 & 2 \\
$\begin{array}{c}\text { 2-Point } \\
\text { discrimination }\end{array}$ & Negative & 0 \\
Likert's scale mean & 2.88 & Negative \\
\hline
\end{tabular}

Student's $t$ value for the above parameters works out to 17.6957 against the table $t$ value of 2.58 for more than 30 degrees of freedom at $p=0.01$. Hence, the observed difference between the two VAS scores is highly significant with $p<0.01$. Mean Likert score is 3.38 for all 50 study subjects. This shows that there have been an overall agreement and no instance of either neutral opinion or disagreement. Thus, any bias in the study is ruled out.

Conclusion: Ultimately, the study shows that administration of heated lidocaine entails very little pain immediately after injection as compared to administration of lidocaine at room temperature.

\section{Pralidoxime in organophosphorus poisoning:} are we using them in the right way?

\section{S. Bahuleyan, V. Rohit, S. Seshadri}

Kasturba Medical College, Manipal, Karnataka, India. shibubahuleyan@yahoo.co.in

Background: Traditionally atropine is the only drug considered useful for organophosphorus poisoning. The role of oximes in organophosphorus poisoning has been debatable. But recent studies have shown that continuous infusion of high dose of oximes shows better outcome when compared to intermittent dosing. Hence, we aimed to assess the effectiveness of the traditional intermittent repeated bolus doses of pralidoxime compared with continuous infusion of pralidoxime in two different dosing regimes.

Method: This was an open-labelled, cross-sectional, nonrandomized observational study. From Oct 2007 to Sept 2008,75 patients were treated at our centre for organophosphorus poisoning. The treatment instituted to each patient was at the discretion of the attending physician, and each of the regimes was uniformly maintained throughout the period of the study. Observations of primary outcome included the number of patients requiring intubation after admission and 
the number of fatalities in each group. Secondary outcomes that were studied included mean dose of atropine used, mean days ventilated, mean days in ICU, incidence of intermediate syndrome and occurrence of pneumonia.

Results: All patients were given atropine and $1 \mathrm{~g}$ loading dose of pralidoxime. Further, depending on the attending physicians, the first group received $1 \mathrm{~g}$ pralidoxime 6th to 8th hourly in intermittent bolus doses. The second group received infusion pralidoxime as $500 \mathrm{mg} / \mathrm{h}$ for $48-72 \mathrm{~h}$, and depending on the clinical response, this was tapered to $250 \mathrm{mg} / \mathrm{h}$ for the next $48 \mathrm{~h}$ and further tapered to $125 \mathrm{mg} / \mathrm{h}$ and stopped. The third group received infusion of $1 \mathrm{~g} / \mathrm{h}$ for $48 \mathrm{~h}$ followed by $500 \mathrm{mg} / \mathrm{h}$ for $48 \mathrm{~h}$ and then tapered to $250 \mathrm{mg} / \mathrm{h}$ for the next $48 \mathrm{~h}$. Outcome analysis showed that patients on continuous infusion of pralidoxime had significantly improved recovery rate $(96 \%$ in the $500-\mathrm{mg} / \mathrm{h}$ group, $90 \%$ in the $1-\mathrm{g} / \mathrm{h}$ group and $64.7 \%$ in the intermittent injection group; $p=0.001$ ). Mean total atropine requirement was lower in the pralidoxime infusion group than in the intermittent group. Mean days on ventilator in the intermittent group was $3.56+$ 4.29 days vs. $1.38+2.23$ days for the $500-\mathrm{mg} / \mathrm{h}$ group vs. $3.26+4.81$ days for the $1-\mathrm{g} / \mathrm{h}$ group; $p=0.002$. No adverse reactions were observed to pralidoxime in such high doses. Conclusions: A continuous infusion of pralidoxime in a dose of $500 \mathrm{mg} / \mathrm{h}$ for $48-72 \mathrm{~h}$ followed by tapering infusion rate based on clinical response reduces morbidity and mortality in moderate to severe cases of acute organophosphorus pesticide poisoning.

\section{Assessing competency of the Broselow-Luten pediatric resuscitation tape: a prospective analytical study of 5,816 South Indian children}

\section{S. Cattamanchi, T.V. Ramakrishnan, Vilvonathan}

Sri Ramachandra Medical College \& Research Institute, Porur, Chennai, Tamil Nadu, India. c.srihari@gmail.com

Background: There are no standardized methods in use for rapid weight estimation in children admitted for acute pediatric emergencies in Chennai, India. The Broselow tape has shown to improve accuracy in weight prediction and eliminates the need for memorization and calculation in emergency situations.

Aims: The aims of this study were (1) to determine the accuracy and usefulness of the Broselow pediatric emergency tape in the Indian pediatric population, (2) to calculate the standard deviation of weight from Broselow pediatric emergency tape for the Indian population, and (3) to devise a new pediatric emergency tape if needed based on the Broselow-Luten tape.

Methods: A prospective, cross-sectional study of 15,000 South Indian children in three weight-based groups of $>10$,
$10-18$, and $<18 \mathrm{~kg}$. Children aged 12 years and under were included in this study. The data were collected from children attending OPDs and EDs in tertiary care hospitals in Chennai and also from schools of all four southern states of South India. Age, sex, height, actual weight, and Broselow weight were recorded in a form between May 1st, 2008 and October 31st, 2008. Measured weight was compared to Broselow-predicted weight and percent difference was calculated. A cross-validated correction factor was derived by non-linear regression. Analysis was done using SPSS ver. 15.0.

Results: A total of 5,816 subjects were included till date, $42.6 \%$ were females, median age of 7.066 years. Weights ranged from 2.25 to $36.0 \mathrm{~kg}$ with a mean of $11.61 \mathrm{~kg}$. Subjects were divided into the three weight-based groups comprised of $1,648(<10 \mathrm{~kg}), 2,356(10-18 \mathrm{~kg})$, and 1,812 $(>18 \mathrm{~kg})$ children. The mean percentage differences were $2.4 \%, 13.3 \%$, and $17.9 \%$ for each weight-based group. The Broselow color-coded zone agreement was $65.8 \%$ in children weighing less than $10 \mathrm{~kg}$, but only $45.3 \%$ in the 10 - to $18-\mathrm{kg}$ group and $32.5 \%$ in the $>18-\mathrm{kg}$ group.

Conclusions: The Broselow tape overestimates weight by more than $10 \%$ in Indian children $>10 \mathrm{~kg}$, increasing risk of medical errors due to incorrect dosing or equipment selection. The need for a modified Indian pediatric emergency tape based on Broselow-Luten color coding is the need of the hour.

\section{Randomized double-blinded control trial comparing the role of ketamine plus morphine versus ketamine alone in providing analgesia for patients with isolated limb injuries}

\section{T. A. Thangalvadi, R. Roy, T. V. Ramakrishnan, P. Kaur}

Sri Ramchandra Medical University, Chennai, Tamilnadu, India. Indian council of medical research. Chennai, Tamilnadu. tausifat@yahoo.com

Aims and objectives: In the management of pain in trauma, ketamine plus morphine provides better analgesia when compared to morphine used alone. Also, the dose of morphine required, when used with a small dose of ketamine, is less thereby reducing the risk of adverse effects associated with it.

Methods: A prospective, randomized, double-blinded, placebo-controlled, interventional trial was done at a tertiary care university hospital in Chennai, India over a period of 3 months from 1st August 2008 to 31st October 2008. All trauma patients between the age group of 18 to 60 years with isolated limb injuries and visual analog pain score of $6 / 10$ or more were included in the study. Hemodynamic instability, any evidence of head injury or 
raised ICP, history of bronchial asthma, pregnancy, or documented allergy to opioids or ketamine was excluded. Written consent was taken from all patients.

Methodology: All patients included in the study received an initial dose of ketamine or placebo $(0.02 \mathrm{mg} / \mathrm{kg})$, based on the color they fell into, after recording vitals and VAS score, followed by a dose of morphine $(0.05 \mathrm{mg} / \mathrm{kg})$. VAS score and vitals were recorded 15 and $30 \mathrm{~min}$ after drug administration. Second dose of morphine was given $15 \mathrm{~min}$ if VAS score was $>3 / 10$. VAS $<3 / 10$ or total pain relief and total dose of morphine required at the end of $30 \mathrm{~min}$ were taken as outcome measures.

Results (interim results): Fifty patients were included in the study. Male predominance of $98 \%$ was noted. Median age was 30.7 years, ranging from 18 to 58 years. At baseline, initial mean VAS scores were $7 \pm 0.8$ in the intervention group and $7.3+0.5$ in the control group. At $15 \mathrm{~min}$, mean VAS scores in the control group were $4.9 \pm 1.4$ and $2.8 \pm 0.8$ in the intervention group. At $30 \mathrm{~min}$, mean VAS scores further reduced to $3.6 \pm 0.9$ in the control group and two in the intervention group. Mean Likert's scale of ease of use was 7. Two patients have hypertension in the intervention group and one had sedation score of 2 in the intervention group.

Conclusion: There is a significant difference in pain relief in patients receiving morphine plus ketamine when compared to patients receiving morphine alone. Pain relief is better and achieved at a faster rate $(3 / 4$ th of the patients in the intervention arm had pain relief at $15 \mathrm{~min}$ versus none in the control arm). There was no significant difference in the risk of adverse effects between the two groups.

\section{Poster Presentations}

\section{Boxer's splint: an innovation}

S.T. Varghese, P.N. Pandian, R. Nithyanandham, V.P. Chandrasekaran

Vinayaka Mission Kirupananda Variyar Medical College Hospital, Vinayaka Mission University, Salem, India

Background: It is a known fact that patients who are restless, hypoxic, conscious but disoriented need continuous care and proper handling in ambulance, emergency, ICU, and wards. In spite of meticulous care, it is not uncommon to have preventable complications like pulling out of ETT, ICD, Foley's catheter, Ryle's tube and IV access. Of these, the first two listed are life-threatening and the others carry high risk of morbidity and mortality. In order to prevent these complications, we have introduced boxer's splint in our institute. Boxer's splints are made by placing a cotton ball into the palm and covering the hand with a cotton cloth bag.
Aim: The aim of the study was to evaluate the utility of boxer's splint in patients with altered sensorium.

Methods: This was a prospective study conducted in our university hospital from August 2006 to August 2008. All the patients who were in altered sensorium in ER and ICU were included in this study. Patients who had injuries in the hand and normal mentation were excluded. Every alternative patient included was put on boxer's splint and the rest had only hand restraints. All patients were followed till recovery and complications relating to the study were noted. Data were collected from 370 patients of which 185 were in the control group and the rest were included in the study group. Results: None of the patients in the study group had any complications related to pulling out of tubes or lines whereas clinical data obtained from the control group showed incidences such as self-extubation (14, 7.5\%), pulling of Foley's catheter (50, 27\%), Ryle's tube (26, 14\%), IV access removals (70, 37\%), ICD tube (10, 5.4\%), and drain (15, 8.1\%).

Conclusions: Boxer's splint reduces complications related to dislodgement of tubes and lines in patients with altered sensorium.

\section{ATLS awareness: the immediate necessity for emergency rooms in Salem}

B. Vasanthi, G. Anand Kumar, V.P. Chandrasekaran, P.N. Pandian

Vinayaka Missions Annapoorna College of Nursing, Salem, Tamilnadu, India

Background: The number of vehicles is increasing at an alarming rate and this has led to more accidents. The number of deaths due to RTAs has also increased drastically. Hence, we wanted to assess the advanced trauma and life support (ATLS) knowledge of emergency personnel.

Aim: With the above background, we decided to evaluate the knowledge of ATLS protocols in the emergency departments of Salem hospitals.

Inclusion criteria: This study included all personnel working in the emergency room who deal directly with trauma cases. It included consultants from various fields involved in trauma cases. Also included were post-MBBS doctors, nurses, and paramedics from some of the major hospitals in Salem.

Exclusion criteria: We excluded Vinayaka mission subjects as most of them have undergone ATLS training.

Methodology: We decided to evaluate the knowledge of ATLS among these personnel with the help of a questionnaire. The questionnaire consisted of ten simple problems which would be commonly faced within the ER when dealing with a case of trauma. Each question was given 1 mark. We graded 
the marks as excellent ( $>8$ marks), good (5 to 8 marks), and less than 5 as requiring ATLS training ASAP.

Results: We were able to gather data from 103 medical personnel from various hospitals of Salem. This comprised 23 consultants, 33 casualty medical officers, and 47 nursing and paramedic staff. In our study, 79 personnel scored less than 5,17 scored more than 8 , and 7 scored 5 to 8 marks. Only $40 \%$ of consultants, $13 \%$ of nursing and paramedics, and $6 \%$ of CMOs scored 8 or more.

Conclusion: The results of our study clearly establish the immediate necessity for an internationally recognized ATLS awareness program.

\section{Does head CT aid in the diagnosis of syncope in the emergency department?}

\section{Velez, L.A. Serrano, M.F. Bellolio}

Department of Emergency, Medicine University of Puerto Rico, Carolina, Puerto Rico.velez_i@yahoo.com

Objective: Current guidelines do not recommend the routine screening of syncope with advance imaging, but it is still a common practice among emergency physicians. Our goal was to determine the usefulness of head computed tomography (CT) scan aiding in diagnosing the cause of syncope in patients presenting to an academic emergency department.

Methods: This study was a retrospective cohort study of all consecutive patients who presented to a single academic emergency department (ED) with syncope during a 6-month period with documented syncope in the medical record. The primary outcome was abnormal head CT with relevant findings to the cause of syncope.

Results: A total of 183 patients presented to the ED with a diagnosis of syncope during January and July 2004, 13 patients were excluded because they met one or more exclusion criteria. The mean age was 58.5 years and 58\% were female patients. Of the total patients studied, 57 patients had a head CT ordered and among them only three had an abnormal head CT. When comparing the group with head CT and the group without head CT, we found the mean age among the head CT group to be 68 years vs. 58.5 years, a 10-year older difference in the head CT group $(p=0.034)$. In the head CT group, $56 \%$ of the patients have non-witness syncope and $44 \%$ patients have witness syncope $(p=0.0448$ OR 2.0; 95\% CI 1.01-3.8). In the head CT group, we have $63 \%$ of patients with first time syncope vs. $37 \%$ with a prior episode ( $\mathrm{p}=0.0076$; OR 2.5; 95\% CI 1.3-5.0).

Conclusions: Head CT still is commonly used in syncope patients. Older age, not having a prior history of syncope, and having non-witness syncope predispose a patient to have a head CT ordered, although no change in outcome was found. This study will support the evidence that head
CT for syncope in the absence of focal neurologic findings and significant head trauma may not be necessary and does not aid in the clinical management. By limiting the use of advanced imaging techniques, we will decrease the overall cost of syncope evaluations.

\section{Strategies to improve survival in road traffic accidents}

\author{
M.K. Saxena, S. Sahoo, B.N. Jena
}

Emergency management \& Research Institute Hyderabad, Andhra Pradesh, India. mukul_saxena@emri.in

Background: Road traffic accidents are a leading cause of death and disability, especially in younger and productive group of a population. It is ironic since most of these deaths and disabilities are preventable.

Aims: The aims of the study were to study various risk factors contributing to survival as a measure of outcome, analyze their significance, and formulate strategies that can result in improved outcome results.

Methods: This is a retrospective study of accidents handled by the Emergency Management \& Research institute in 2007. A total of 9,269 cases of accidents involving 12,649 victims were studied with regard to epidemiological variables and certain other variables which have a correlation with survival as a measure of outcome. The statistical methods used were chi-square test and correlation studies.

Results: The risk factors that were identified are as follows: age (18-37 years most vulnerable), anatomical area (head and neck injuries most critical), and type of collision (head on collision has the worst prognosis).

Driving under the influence of alcohol and lower socioeconomic group people were found to be more susceptible to accidents. A variable relationship was noted as regards accidents affecting different age groups and time of accident. A time of accident and type of vehicle relationship was also noted.

Analysis of the abovementioned risk factors was incorporated in formulating certain strategies which can improve survival status in road traffic accidents. The strategies would have to be implemented at different levels, i.e., at the level of reducing response time; interventions during pre-hospital setting; triage, considering inter-facility transfers; implementation of safety rules; traffic organization, through different departments like the police; ambulance segmentation policies for effective pre-hospital care; and using social marketing practices and a comprehensive trauma management policy involving healthcare providers, the state, and NGOs.

Conclusion: In summary, road traffic accidents are considered to be a complex conglomerate of various risk factors which need to be aggressively addressed by different strategies discussed in this paper. 
Audit of protocol for evaluating fever in adults with no localizing signs presenting to the emergency department

\section{Dr Sudhagar}

Sundharam Medical Foundation, Chennai, Tamilnadu, India

\section{YOUNG RESEARCHER AWARDS}

\section{Post graduate category}

\section{Outcome in a Case Series on Tetanus}

P. Mohanasundaram, P.N. Pandian, R. Nithyanandhan, V.P. Chandrasekaran

Vinayaka Mission Kirupananda Variyar Medical College Hospital, Salem, India.

Background: Tetanus remains a major problem in farmers and low socioeconomic group of people. We report a case series of tetanus. Early diagnosis with meticulous and aggressive management is necessary to avoid complications and save patients. The experience from this reveals that there is a lot of hesitation to accept tetanus cases in many hospitals and a better outcome is possible if it is treated appropriately. Aims: The aims of the study were to create an awareness on tetanus and its management and to encourage practitioners to admit and treat with confidence and good outcome.

Case series: Seven cases of tetanus were treated in our hospital. No significant age factor for acquiring the disease was found. They were either farmers, scavengers, or bread winners of the family. Common causes were thorn pricks, contaminated wounds, burns, wounds, and inadequate immunization. All patients had neck rigidity, lock jaw, rigidity of limbs, exaggerated deep tendon reflexes, opisthotonus, and further progression. These patients were diagnosed early and aggressive management was initiated in the emergency room (ER). Airway and breathing was taken care of. Elective tracheostomy was done at the ER.

All patients were paralyzed with neuromuscular blocking agents and supported with mechanical ventilation and they all received midazolam and fentanyl infusions. All were started on tetanus toxoid.

Only two patients received TIG due to financial constraints. They were admitted to the ICU with appropriate care. Metronidazole was initiated for all 8th hourly for 21 days. Every second day, paralysis was stopped and reassessed and neuromuscular blocking agent was continued until spasm was relieved. Dantrolene sodium was started for five patients. Most of them had autonomic instability and were managed with beta blockers and clonidine. Nutrition plays an important role with supplementation of appropriate calories. Other complications during treatment were managed appropriately. All the patients received supportive measures like bed sore prevention, nosocomial infections prevention, DVT prophylaxis, stress ulcer prophylaxis, oral hygiene, fluids and electrolytes, and physiotherapy. They were subsequently weaned from the ventilator.

Results: Six out of seven cases walked out of the hospital with complete recovery. One case which presented late to our hospital with uncontrolled autonomic instability, renal failure, and rhabdomyolysis died within $24 \mathrm{~h}$ of admission. Only two cases received tetanus immunoglobulin of which one died due to late presentation with complications. The remaining five cases who survived showed no significant difference in the duration of stay in the hospital and outcome. Dantrolene was used for five out of the seven cases who showed no significant difference in the duration of stay in the hospital and outcome. By the end of 3 weeks, the patients were able to walk short distances with assistance of physiotherapist and at approximately one month patients were discharged home with complete recovery and till date all are still surviving.

Conclusion: Tetanus is a curable disease if early diagnosis, planning, counseling of the family, and prompt and aggressive preliminary interventions are initiated at the ER and continued till recovery.

\section{Under-graduate category}

\section{Role of activated charcoal in prehospital poisoning care}

T.M. Sathish, S. Narayana, S.S. Kumar, S. Chandrasekaran

Vinayaka Mission University, Salem, India

Background: The emergency department at Vinayaka Mission reveals a hallmark in handling the poisoned victims by using activated charcoal for plant poisoning.

Aim: The aim of the study was to assess the efficacy of early charcoal administration at the community level among poisoned victims.

Methods: One thousand packets of activated charcoal, each weighing approximately $50 \mathrm{~g}$, were prepared along with the instruction cards attached regarding the directions, indications, and contraindications in the local language. These packets were distributed free of cost to the local alternative physicians and to the medical stores/pharmacies, petrol stations, tea stalls, police station, grocery shop, and to the village Panchayat office, and they were instructed to deliver them free of cost to those who approached them with history of poisoning, either for themselves or for any person they know after noting down their 
address. Propaganda regarding availability of a new "life saver medication" for poisoning patients, especially for plant and pesticide poison was done in the villages with banners and pamphlets and in the places where these "life saver" drug is available.

Results: After 6 months of propaganda and regular followup of the places, it was found that a total of 22 packets have been distributed, and out of which we had contact details of 19 members. They were contacted and enquired about the type of poison and duration after which the charcoal was given and the current status of the patient.

Out of 19 victims, eight were due to plant poisoning, nine due to pesticide/insecticide poisoning, and two due to sani powder poisoning.

Of these, all eight plant poisoning victims, seven pesticide poisoning victims, and two sani powder poison victims have survived, one victim of insecticide died, and one refrained from providing any information requested by us due to social stigma.

Conclusions: Early activated charcoal administration in the pre-hospital setup is useful in saving valuable life.

\section{SPECIAL MENTION AWARDS}

Evaluation of severity scoring systems in ICU care: APACHE II and SAPS II

\section{A.B. Saji, A.S. Kumar, T.V. Ramakrishnan}

SRMC, Chennai, Tamil Nadu, India.drsajiab@rediffmail.com

Background: In recent years, several scoring systems have been developed to describe the severity of illness, to establish the individual prognosis, and to group the intensive care unit (ICU) patients by predicted risk of mortality. We compared the mortality predictions of the Acute Physiology and Chronic Health Evaluation (APACHE II) and Simplified Acute Physiology Score (SAPS II) in adult patients admitted to multi-disciplinary ICUs of a tertiary care university hospital, Chennai, India. Aims: A prospective, comparison study was designed to evaluate and compare the ability of APACHE II and SAPS II scoring systems and to predict the mortality in adult patients admitted in multi-disciplinary ICUs of a tertiary care university hospital in India.
Methods: The study presents information on randomly selected ICU patients, from 1st July 2008 till date. Patients above 18 years of age and those who stayed in the ICU for more than $24 \mathrm{~h}$ were included in the study. Patients of 18 years of age and less and post-cardiac arrest patients were excluded from the study.

A preformatted questionnaire was used to collect all the information necessary to compute the APACHE II and SAPS II scores, demographics, and hospital outcome. APACHE II and SAPS II scores were calculated in accordance with the original methodology, using the worst physiologic values during the first $24 \mathrm{~h}$ of ICU admission.

Predicted mortality was calculated using original regression formulas. Standardized mortality ratio (SMR) was computed with 95\% confidence intervals (CI). Discrimination was evaluated by calculating the area under the receiver operating characteristic curves (ROC AUC).

Results: Predicted mortality by both systems was different from the actual mortality, probably due to the short duration of the study and small sample size [SMR for APACHE II is $0.34(0.25-0.43)$ and for SAPS II $0.35(0.245-0.435)]$. Discrimination was better for SAPS II (ROC AUC 0.67) than APACHE II (0.69).

Conclusions: Overall mortality prediction of APACHE II and SAPS II scoring systems, estimated by standardized mortality ratio, was not accurate for our studied ICU population. Discrimination was better for SAPS II when compared to APACHE II.

A study to compare the level of professional life stress experienced by nurses in emergency departments and in general wards and to develop guidelines to overcome stress

Anna

Shanmuga College of Nursing, Salem, Tamilnadu, India

Reduction of bloodstream MRSA using alcohol-based body wash

Dr. Vetrivel Ramar

Apollo Hospital, Bangalore, India 